\title{
Caracterización de los principales tipos de mármol extraídos en la comarca de Macael (Almería, sureste de España) y su importancia a lo largo de la historia
}

\author{
R. Navarro ${ }^{(1,5)}$, A. S. Cruz ${ }^{(2)}$, L. Arriaga ${ }^{(3)}$ y J. M. Baltuille ${ }^{(4,5)}$ \\ (1) Instituto Geológico y Minero de España (IGME). Oficina de Granada. Urb. Alcázar del Genil, 4 Edif. Zulema, Bajo. 18006 Granada, Spain. \\ rafanavarro74@gmail.com \\ (2) Cestema Ingeniería Minera. Ctra. Olula-Macael km. 1,7. 04867 Macael, Almería, Spain. \\ anasol.cruz@cestema.es
}

(3) Asociación de Empresarios del Mármol de Andalucía (AEMA). Ctra. Olula-Macael km. 1,7. 04867 Macael, Almería, Spain. comunicacion@macaelmarmol.com

(4) Departamento de Infraestructura Geocientífica y Servicios. Instituto Geológico y Minero de España (IGME). Ríos Rosas, 23. 28003 Madrid, Spain.

jm.baltuille@igme.es

(5) Red CONSTRUROCK (Red de la Piedra Natural y su relación con el Patrimonio Histórico-Monumental y la obra nueva, en el ámbito del Estado español). Ríos Rosas, 23, 28003 Madrid. Spain.

\section{RESUMEN}

El mármol de Macael es una de las rocas ornamentales más importantes de España. Se ha empleado para la realización de elementos tanto decorativos como de construcción, especialmente la variedad “Blanco Macael." Las evidencias de su uso se remontan a más de 4000 años. Uno de los ejemplos más destacados es el Patio de los Leones en la Alhambra (Granada), pero también se pueden observar algunos elementos en el anfiteatro romano de Mérida (Badajoz), en Itálica (Sevilla), en el Palacio de Medina Azahara (Córdoba) o en el Palacio Real de Madrid, entre decenas de palacios o iglesias por todo el país. En el presente trabajo se describe la utilización que ha tenido este material a lo largo de la historia y se indican las principales características y propiedades de las diferentes variedades: "Blanco Macael", "Gris Macael", "Anasol” (mármoles calcíticos) y "Amarillo Macael" (mármoles dolomíticos). Todos ellos presentan unas cualidades mineralógicas y físico-mecánicas que los hacen apropiados para prácticamente todos los usos. Los mármoles calcíticos presentan porosidad, absorción y densidad más bajos que los dolomíticos, mientras que los mármoles dolomíticos presentan mayor resistencia a la compresión, una resistencia a la flexión similar, algo inferior, y una resistencia a los anclajes inferior que los calcíticos. Los valores de resistencia a la abrasión son también mejores en los dolomíticos, mientras que la resistencia al deslizamiento es similar en todos los casos. En cuanto a la alteración los dolomíticos, con la excepción de la variedad Amarillo Triana, se alteran más en los ciclos de hielo/deshielo. En lo referente a la alteración por cristalización de sales, la que menos alteración presenta es la variedad Blanco Macael Río (calcítica), pero en conjunto, los mármoles dolomíticos presentan menos alteración que los calcíticos. En cuanto al estado del sector de la piedra en la región, hay que destacar que a pesar de una bajada en las ventas en el mercado nacional de un 60\%, las exportaciones en el 2012 se incrementaron un $49 \%$ respecto al año anterior. Los datos presentados permitirán incrementar el conocimiento acerca de uno de los materiales de construcción más empleado en nuestro país de cara a optimizar su uso por parte de las empresas comercializadoras y consumidoras.

Palabras claves: Macael, mármol, patrimonio arquitectónico, roca ornamental, propiedades tecnológicas.

\section{Characterization of the main types of marble extracted in the area of Macael (Almeria, southeastern Spain) and its historical importance}

\author{
ABSTRACT
}

The marble from Macael is one of the most important ornamental stones found in Spain. It has been used not only as a building material but also as a decorative element, especially the type known as "White Macael." There is evidence of its use dating back over 4000 years. One of the most prominent examples is the 
Navarro, R., et al., 2017. Caracterización de los principales tipos de mármol .... Boletín Geológico y Minero, 128 (2): $345-361$

Courtyard of the Lions in the Alhambra (Granada), but it is also possible to see some elements in the Roman amphitheatre of Mérida (Badajoz), in Italica (Sevilla), in the palace of Medina Azahara (Córdoba) and in the Royal Palace of Madrid, amongst dozens of palaces and churches throughout the country. In this study we describe the use that this material has had throughout history and the main features and properties of the different varieties of "White Macael", "Grey Macael", "Anasol" (calcite marbles) and "Yellow Macael" (dolomitic marbles). They all have mineralogical, physical and mechanical features that make them suitable for almost all uses. Calcite marbles have porosity, absorption and density values lower than dolomite marbles, whilst the latter have more resistance to compression, similar flexural strength under concentrated load, and lower resistance to breaking load at dowel hole than the calcitic marbles. The values of abrasion resistance are also better in the dolomite marbles, whilst the slip resistance is similar in all cases. As far as decay is concerned, the dolomitic marbles, with the exception of the variety Amarillo Triana, suffer more in the frost resistance test but less by the action of salts, with the exception of the White Macael Rio (calcitic) which is the variety that has less alteration. Regarding the status of the stone sector in the region, it is noteworthy that, despite a $60 \%$ drop in sales in the domestic market, exports in 2012 increased by $49 \%$ compared to the previous year. The data we present provides increased knowledge of one of the most common building materials in our country with the aim of optimizing its use by marketing companies and by consumers.

Keywords: architectonic heritage, Macael, marble, natural stone, technological features.

\section{ABRIDGED ENGLISH VERSION}

\section{Introduction}

Natural stone is one of the oldest construction materials used by man. Most of the constructions since ancient times have used stone as a basic material. The Egyptians were the first to exploit natural stone in an organized and systematic way in about 2600 B.C. (Harrel 2003). But where the use of marble as ornamental stone was widespread was in ancient Greece and in the Roman Empire in the $7^{\text {th }}$ century B.C. It was an indication of social status (López-Jimeno 1995). Since then, marble has become widely used throughout many cultures.

The marble from Macael is one of the most important ornamental stones used in Spain throughout history, especially from the heritage point of view. Its exploitation began more or less continuously in the $11^{\text {th }}$ century (Pastor-Medina, 1990), although there is evidence of its use more than 4,000 years ago (Grima, 1994). From the beginning of the $20^{\text {th }}$ century, these materials have been exploited continuously using industrial methods (Fig. 1 a, b). This has lead to all kinds of companies in the sector involved in the processes of transformation of the stone, from extraction to final marketing, to become established in the Macael region.

The most typical commercial varieties of marble from Macael include both calcitic and dolomitic marbles. They are known generically as White Macael, Yellow Macael, Grey Macael and Anasol, with many variations and specific names in each mining area, producer or each village.

The aim of this paper is to describe the use that the marble from Macael has had throughout history, the technological features of the main varieties and to analyse the current state of the sector.

\section{Location and geological context}

The "Marble Region" is located in the province of Almería (southern Spain) (Fig. 2). From the geological point of view, the quarries are located in the Nevado-Filabride Complex (CNF), which is the lowest metamorphic complex of the Internal Zones of the Betic Cordillera (Egeler, 1963). The CNF can be subdivided into lower and upper units (Martín-Algarra, et al., 2004), and it is in the upper unit that the Macael quarries are located (Fig. 3). Within this upper unit, we can differentiate two units by tectonic criteria: the unit of Calar Alto, which is the lower, and the unit of Bedar-Macael, which is the upper one where the levels of marble with mining interest are located.

\section{Historical use of marble from Macael}

The first objects made of marble from Macael which we are aware of are from between 2,700 and 2,000 B.C. (Siret and Siret, 2006). They are very basic items such as rings, beads, bracelets, glasses, idols, etc. but it is indicative that these first settlers valued this material. Another important milestone is the discovery of a Phoenician sarcophagus found in Cadiz, dating from the $5^{\text {th }}$ C. B.C. (Almagro-Gorbea et al., 2010). During the Roman period $\left(1^{\text {st }}-2^{\text {nd }}\right.$ C.) exploitation of the quarries was more systematic (Grima, 1994). There is evidence of 
Navarro, R., et al., 2017. Caracterización de los principales tipos de mármol .... Boletín Geológico y Minero, 128 (2): $345-361$

its exploitation in Macael, Lubrín and Cherchos and it was used in numerous tombstones, statues, columns and epigraphic supports (Padilla, 1999). We can see examples of this period in the amphitheatre of Merida,

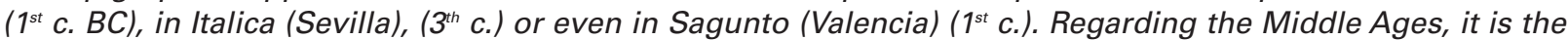
Muslim period when the systematic and planned quarrying really began (Carretero-Gómez, 1995; Sáez-Pérez, 2004; Luque, 2010). At that time Macael marble was marketed under the name "Al Maluki" (the Royal). The first examples of this period are found in places such as the palace of Medina Azahara (Córdoba) (10 ch.) or the Courtyard of the Lions and the Hall of the Two Sisters in the Alhambra (Granada) (14 ${ }^{\text {th }}$ c.). The great proliferation of this material was from the 16th c. The construction of the Monastery of El Escorial is a milestone in the use of marble in the peninsula due to its richness in ornamental stones. Another milestone in the use of marble in Spain was the construction of the Royal Palace in Madrid (18th c.), where Spanish natural stones are used almost exclusively, including marble from Macael. Actually, it is one of the most prestigious building stones in Spain, both nationally and internationally, and is still being used in many buildings worldwide. Some examples are shown in Figure 4, both historical and recent, of the use of different types of marble from Macael.

\section{Materials and methods}

We analysed the following types of marbles: a) White Macael: Río (BMR), Puntilla (BMPu), Polonia (BMPo) and Cañaílla (BMC); b) Yellow Macael: Río (AMR), Triana (AT), Indalo (Al) and Alhambra (AA); c) Grey Macael (GM) and d) Anasol (AS).

Mineralogical analysis was performed by X-ray diffraction (XRD) (Panalytical X'pert-Pro diffractometer) and optical polarized light microscopy (MOP) (Leica DM2500P). Regarding the hydric properties, open and total porosity, bulk density (UNE-EN 1936) and absorption of water at atmospheric pressure (UNE-EN 13755) have been determined. As for the mechanical parameters, we determined the compressive strength (UNE-EN 1926), flexural strength under concentrated load (UNE-EN 12372), breaking load at dowel hole (UNE-EN 13364) abrasion resistance (UNE-EN 14157) and slip resistance (UNE-EN 14231). Regarding the decay test we have carried out the frost resistance test (UNE-EN 12371) and the salt crystallization test (UNE-EN 12370).

\section{Results and discussion}

Macael marble can be classified from the point of view of its mineralogy into calcite and dolomite marble. The first group includes all varieties of White, Grey and Anasol marbles, whilst in the second, the varieties of yellow marble. In Figure 5 we show the macroscopic appearance of polished samples of these kinds of marble.

Varieties of calcitic marbles are mainly composed of calcite, along with other minerals such as mica, quartz, pyroxene, plagioclase and opaque minerals (pyrite, ilmenite), whilst varieties of dolomitic marbles are composed of dolomite, together with other phases such as micas, quartz, and iron oxides and manganese (Fig. 6), in a much smaller percentage. The texture observed in all cases is granoblastic. The grain size is between 1.20 and $2.20 \mathrm{~mm}$ in BMR varieties (Fig. 7a) and GM and between 0.17 and $0.25 \mathrm{~mm}$ in the varieties of AMR (Fig. $7 \mathrm{~b}$ ). The AS has an intermediate grain size compared to the previous varieties.

Regarding the technological properties, Table 1 shows both the main physical and mechanical properties as well as the alterability properties of the studied marbles. It should be noted that calcite marbles have porosity, absorption and density values lower than dolomite marbles, whilst the latter have more resistance to compression, similar flexural strength under concentrated load, and lower resistance to breaking load at dowel hole than the calcitic marbles. The values of abrasion resistance are also better in the dolomite marbles, whilst the slip resistance is similar in all cases. As far as decay is concerned, the dolomitic marbles, with the exception of the variety Amarillo Triana, suffer more in the frost resistance test but less by the action of salts, with the exception of the White Macael Rio (calcitic) which is the variety that has less alteration.

In relation to state of the sector, Spain is currently the seventh largest producer of natural stone after Italy and Brazil, with a production of 5.5 Mt in 2011 (AIDICO, 2012) of which $2.3 \mathrm{Mt}$ corresponds to both ornamental limestone and marble (INE, 2013).

Despite the decline of the sector in recent years which has caused a great reduction in the number of active quarries in the region of Macael, strong international demand for this product has still maintained a high productivity compared to other sectors of the building industry. Table 2 shows the variation of the factory production and exports for the last 10 years. It highlights that production has fallen by almost half in recent years. However, companies, facing the decline of the domestic market, have chosen to promote foreign trade, a fact that has been rewarded with a gradual increase in the volume of exports, notably the year 2012 with an increase of almost 50\% over the previous year. The main countries to which Macael marble is exported are the U.S., Germany, UK, Netherlands, France, Ireland, Portugal, Italy, Japan, Morocco, Arab Emirates, Russia and China. 
Navarro, R., et al., 2017. Caracterización de los principales tipos de mármol .... Boletín Geológico y Minero, 128 (2): $345-361$

\section{Introducción}

La piedra natural es el material de construcción más antiguo, ya que la mayor parte de las construcciones erigidas por el hombre, desde tiempos remotos, han tenido este material como base. Ya desde los primeros albores de la historia, el hombre utiliza la piedra con fines, primero en un sentido mágico-religioso (Stonehenge, menhires, dólmenes, etc.), y posteriormente como piedra de construcción u ornamento (tumbas, sarcófagos, cabañas, pequeñas estatuas, etc.) (López-Jimeno, 1995).

Los egipcios son los primeros en la explotación de la piedra natural de forma organizada y sistemática, hacia el año 2600 a.C. De hecho, el primer mapa geológico del que se tiene constancia, el conocido como papiro deTurín, fue elaborado alrededor del año 1150 a.C., encargado por el rey Ramsés IV (1156-1150 a.C.) para una de las expediciones enviadas para la explotación de canteras en el área de Wadi Hammamat. EI propósito de estas expediciones era obtener bloques de la denominada piedra de Bekhen (bekhen-stone), una arenisca-limolita metagrauváquica, de color grisverdoso, muy apreciada en el antiguo Egipto para la realización de estatuas de los dioses, reyes y gente notable (Harrell, 2003). Pero donde se generaliza el uso del mármol como piedra ornamental e indicativo del lujo es en la Grecia antigua y en el Imperio Romano, hacia el siglo VII a.C., donde se extraen los más bellos mármoles de todo el mundo conocido para la elaboración de todo tipo de elementos (columnas, estatuas, suelos, etc.) (López-Jimeno, op. cit.), siendo un indicativo del estatus social. A partir de entonces el uso del mármol ha sido algo generalizado por todas las culturas.

La caracterización de las propiedades físicas de las rocas ornamentales es un aspecto básico que contribuye a determinar su calidad y que va a condicionar su durabilidad. Las propiedades físicas más importantes que van a definir su utilización como roca ornamental son el sistema poroso, el comportamiento hídrico y las propiedades mecánicas (Benavente, 2006).

Con respecto a los mármoles, los estudios sobre su comportamiento como roca de construcción son muy numerosos, habiendo cientos de estudios sobre las características petrográficas, hídricas, de porosidad, resistencia mecánica, etc. (Doehne y Price, 2010).

El mármol de Macael es una de las rocas ornamentales que mayor importancia han tenido en España a lo largo de la historia, especialmente desde el punto de vista del patrimonio. Es por ello, que la mayor parte de los estudios realizados hasta la fecha se hayan centrado en determinar las principales cau- sas de deterioro (Bello et al., 1992; Luque, 2010; Luque et al., 2011a, 2011b y 2013; Rodríguez-Gordillo y SáezPérez, 2006 y 2010; Sáez-Pérez, 2004; Sáez-Pérez y Rodríguez-Gordillo, 2009). No obstante hay que indicar que en estos trabajos apenas se detallan sus características físicas y/o mecánicas y no están enfocados en establecer las características técnicas de estos materiales para construcción, como se expone en este trabajo.

La explotación del mármol de Macael comenzó de forma más o menos continuada en el s. XI (PastorMedina, 1990), aunque hay constancia de su empleo desde hace más de 4000 años (Grima, 1994). A partir del s. XX estos materiales se han venido explotando de manera continua y de forma más industrializada (Fig.1 a y b), habiéndose instaurado en la comarca todo tipo de empresas del sector implicadas en todos los procesos de transformación de la piedra, desde la extracción hasta la comercialización final.

Las variedades comerciales más características del
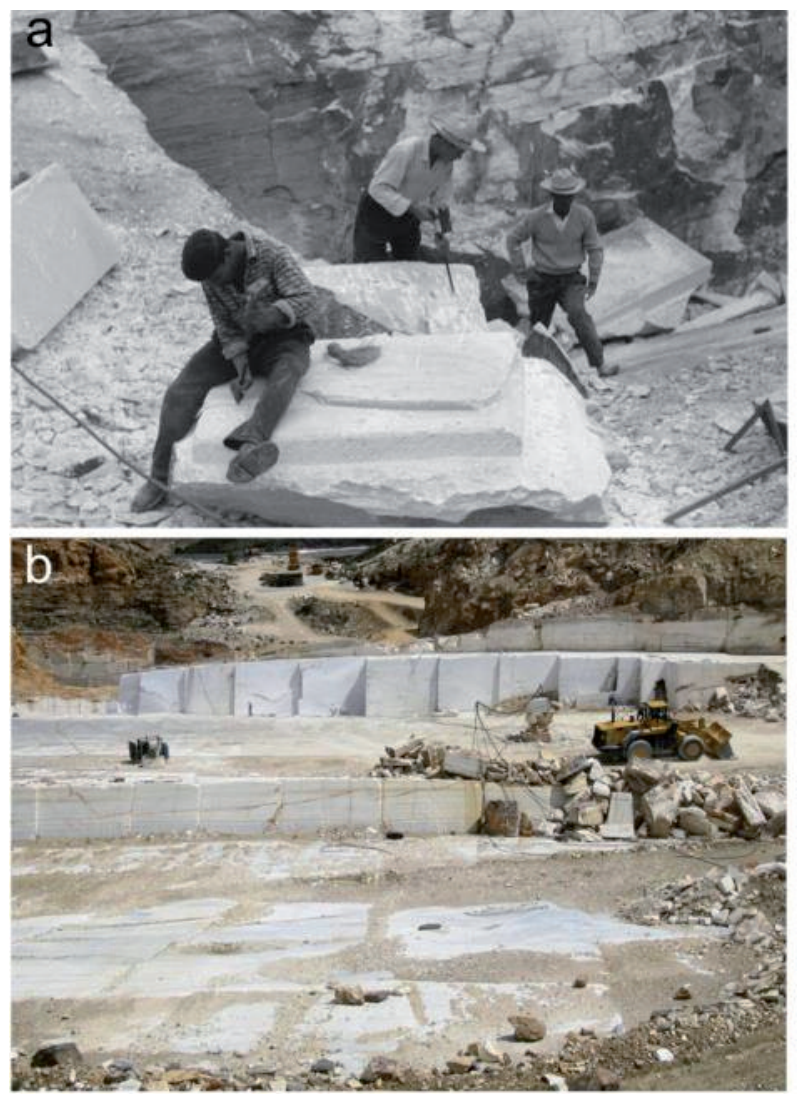

Figura 1. Trabajos de explotación en las canteras de Macael: (a) métodos manuales de recorte de bloques en los años 50 del s. XX (foto: archivo AEMA); (b) trabajo mecanizado en la actualidad.

Figure 1. Exploitation works in the quarries of Macael: (a) manual methods of cutting blocks in the 1950s (20 ch c.) (photo: AEMA), (b) recent mechanized works. 
mármol de Macael incluyen tanto mármoles calcíticos como dolomíticos. Son las conocidas genéricamente como Blanco Macael, Amarillo Macael, Gris Macael y Anasol, con multitud de variaciones y denominaciones más locales propias de cada zona de extracción, empresa comercializadora o de cada municipio. Este material admite todo tipo de acabados, siendo los más comunes el abujardado, apomazado, envejecido, escarfilado, pulido, serrado o al corte. En los catálogos comerciales es posible encontrar, dentro del grupo del mármol, otra roca que se extrae por la zona de Macael, que es la serpentinita (variedades Verde Macael y Verde Almería). Viene catalogada como mármol verde, a pesar de que sus características mineralógicas, geoquímicas, físicas y mecánicas no se corresponde con un mármol (Navarro et al. 2013). Este hecho puede inducir a confusión y por tanto puede llevar a un mal uso del material (Pereira et al. 2013).

La correcta caracterización de la piedra natural utilizada especialmente en patrimonio monumental es una de las prioridades de "CONSTRUROCK" (Red de la Piedra Natural y su relación con el Patrimonio Histórico-Monumental y la obra nueva, en el ámbito del Estado español) (Baltuille et al. 2012) y del "Heritage Stone Task Group", grupo internacional de trabajo vinculado a la IUGS a través de la comisión C10 (Building Stones and Ornamental Rocks) de la International Association of Engineering Geology (IAEG) (Cooper et al., 2013), que nacen con el fin poner de manifiesto la importancia del adecuado conocimiento de las rocas ornamentales empleadas en nuestro entorno para una mejor optimización de los recursos, especialmente en el ámbito del patrimonio.

El objetivo de este trabajo es describir el uso que ha tenido el mármol de Macael a lo largo de la historia. En él se exponen las características tecnológicas de las principales variedades del mármol y se hace un análisis del estado actual del sector del mármol en Macael, lo que es fundamental para su adecuada utilización.

\section{Localización y contexto geológico}

La denominada "Comarca del Mármol" se localiza en la provincia de Almería (sur de España). Los municipios en los que se centra la mayor producción son los de Macael, Olula del Río, Fines, Cantoria, Líjar y Purchena, aunque otros municipios del Valle de Almanzora como Albánchez, Albox, Arboleas,

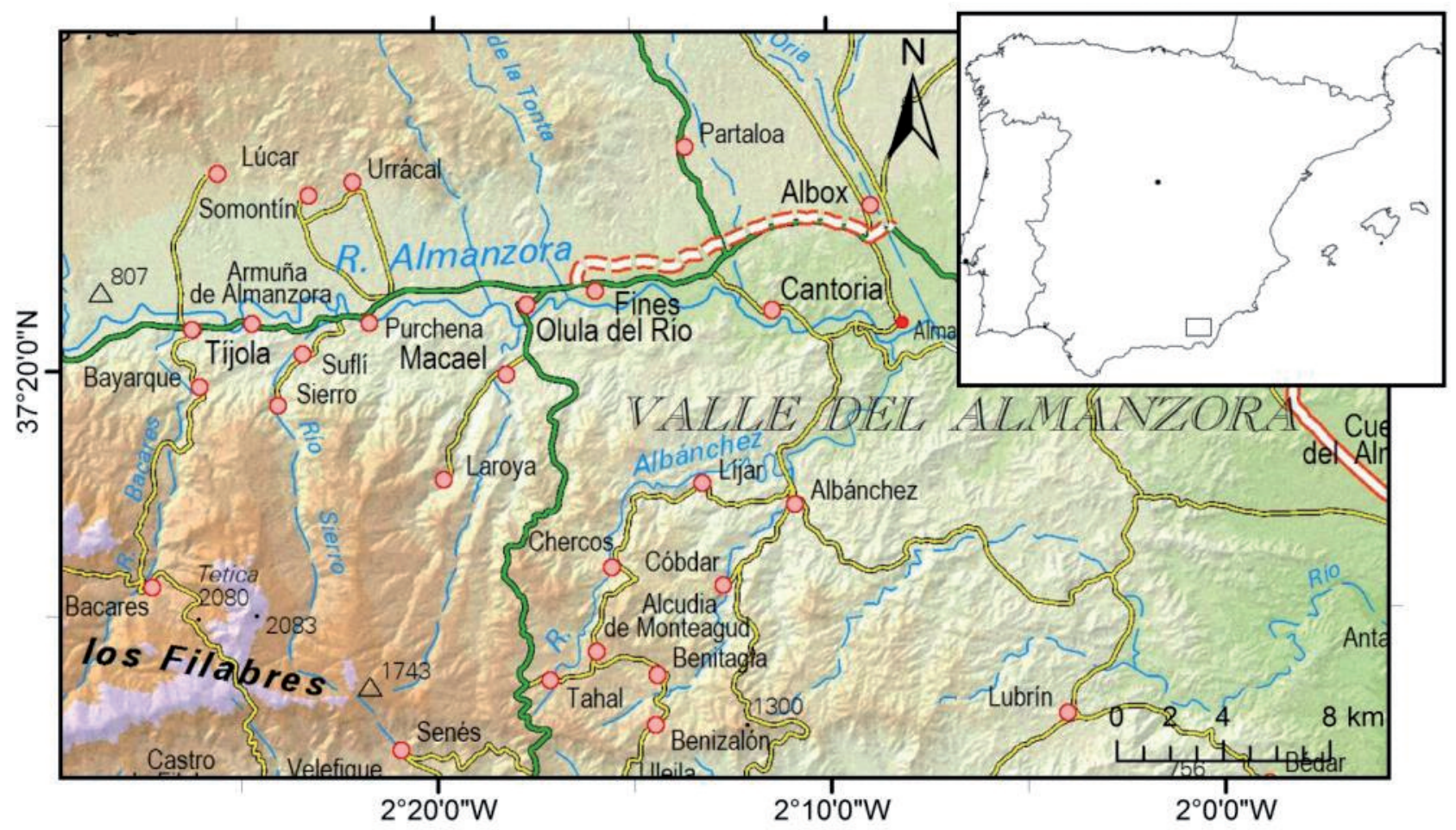

Figura 2. Localización de la "Comarca del Mármol".

Figure 2. Location of the "Marble region". 
Chercos, Cóbdar, Laroya, Lubrín y Zurgena, basan también una parte muy importante de su actividad económica en el aprovechamiento de este recurso. En la Fig. 2 se muestra la situación general del área donde se centran las principales explotaciones mineras de mármol.

Desde el punto de vista geológico, las canteras de Macael se localizan en el Complejo Nevado-Filábride (CNF), que constituye el complejo metamórfico más bajo de las Zonas Internas de la Cordillera Bética (Egeler, 1963). El CNF, a pesar de que no hay un consenso generalizado acerca de las unidades que lo forman, se puede subdividir, por criterios tectónicos, en unidad inferior y superior (Martín-Algarra, el al., 2004), siendo en la superior donde se ubican las canteras de Macael (Fig. 3). La edad de los materiales que forman este complejo va desde el Precámbrico hasta el Jurásico. En cuanto a la edad del metamorfismo, aunque hay evidencias de metamorfismo prePérmico en los materiales más antiguos, se produce principalmente durante la orogenia Alpina (Cretácico Superior-Mioceno) (Martín-Algarra, et al., op cit).

Dentro de la Unidad Superior, García-Dueñas et al. (1988) y Martínez-Martínez et al. (2002), diferencian, por criterios tectónicos, dos unidades:
- Unidad de Calar Alto. Es la unidad tectónicamente inferior, y está compuesta por esquistos grafitosos y cuarcitas, esquistos claros y metasamitas y mármoles calcíticos y dolomíticos

- Unidad de Bédar-Macael. Es la unidad superior y es más heterogénea, compuesta por rocas metamórficas de grado medio, que incluyen mármol calcítico, serpentinitas, micaesquistos de color claro, intercalaciones de mármoles dolomíticos amarillentos y mármoles grises tableados, y gneises bandeados. El material de mayor interés son los mármoles blancos, cuya potencia se estima en unos $40 \mathrm{~m}$, aunque toda el área se encuentra afectada por una deformación importante, con pliegues muy cerrados lo que produce variaciones importantes en el espesor de las capas. Dentro de la Unidad Bédar-Macael se diferencian las siguientes formaciones:

- Formación las Casas: se pueden distinguir dos secuencias litológicas: una compuesta predominantemente por rocas carbonatadas (mármoles calcíticos y dolomíticos) de colores blancos, azules, amarillos y marrón oscuro y la otra por esquistos cuarcíticos.

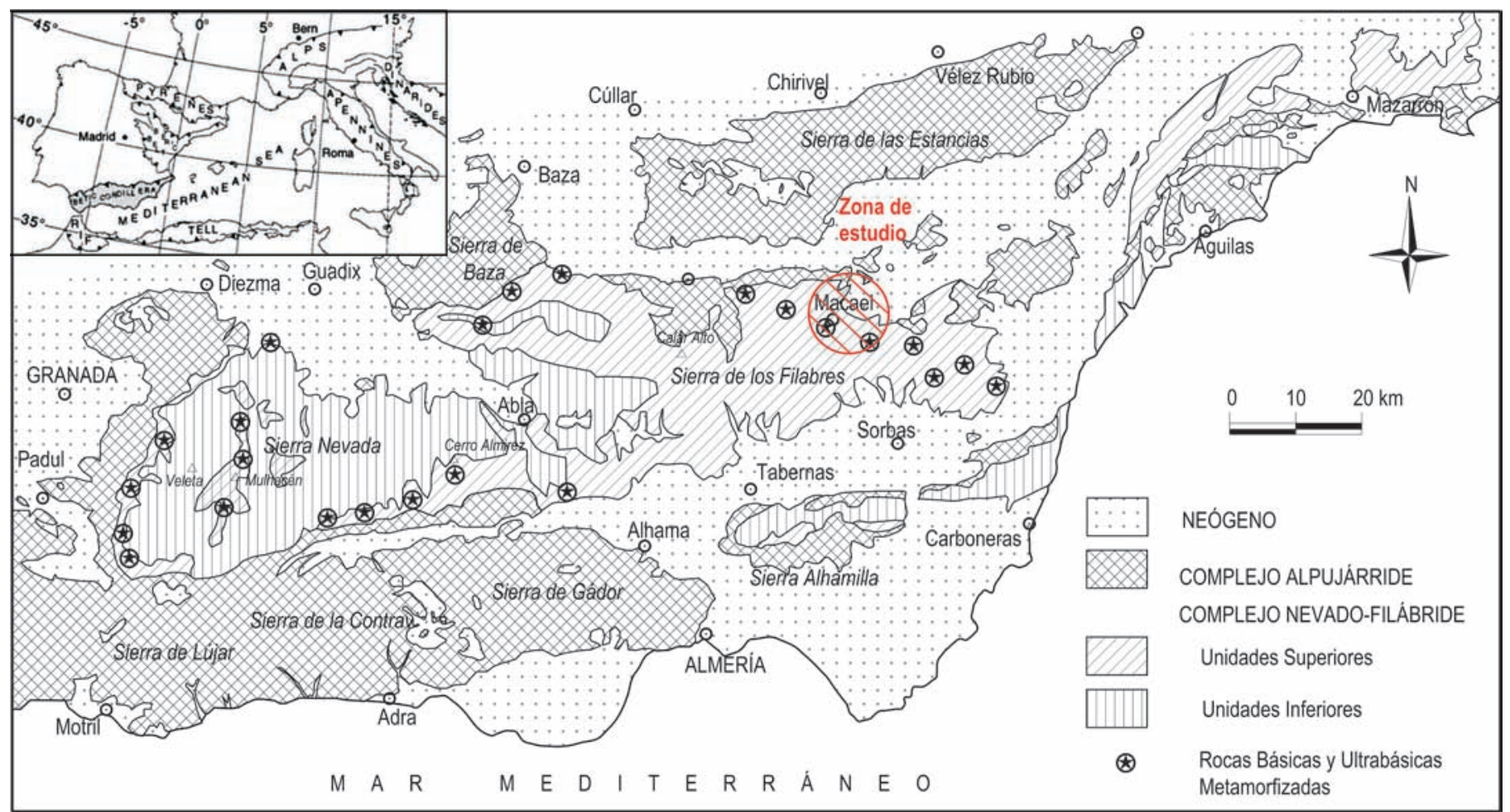

Figura 3. Encuadre geológico de la zona de estudio (modificado de Martín-Algarra et al., op.cit.).

Figure 3: Geological setting of the studied area (modified from Martín-Algarra et al., op.cit.). 
Las rocas carbonatadas se encuentran predominantemente en la parte más alta y más baja de la formación. Es a la que pertenecen casi todos los niveles de mármol de importancia comercial en la comarca (Kampschuur, 1975).

- Formación Huertecicas: alternancia de mármoles con micaesquistos, brechas tectónicas y yesos. Las brechas aparecen como masas irregulares entre las rocas carbonatadas no brechificadas (Kampschuur, op. cit.; García-Dueñas et al., op. cit.; MartínezMartínez et al., op. cit.).

- Formación Tahal: micaesquistos, gneises albíticos y cuarcitas, con alguna intercalación marmórea de importancia, más abundantes hacia el techo (García-Dueñas et al., op. cit.; Martínez-Martínez et al., op. cit.).

- Formación Nevada: alternancia de micaesquistos grafitosos con granates y cuarcitas, con niveles de gneises con turmalina y rocas piroxénicas verdes entre los que se intercalan rocas carbonatadas grisáceas que no exceden de $2 \mathrm{~m}$ de potencia (Kampschuur, op. cit.).

\section{Uso histórico del mármol de Macael}

Las primeros objetos realizados con mármol de Macael de los que se tiene conocimiento son de entre 2700 y 2000 años a.C. (Siret y Siret, 2006). Son elementos muy básicos, como anillos, cuentas de collar, pulseras, vasos, ídolos, etc., pero indican que estos pobladores valoraban ya este material. Otro hito importante es el descubrimiento de unos sarcófagos fenicios encontrados en Cádiz, uno en 1887 (sarcófago masculino) y otro en 1980 (sarcófago femenino), que datan del s. V a.C. (Almagro-Gorbea et al. 2010).

Durante el periodo romano (s. I-II) su explotación comienza a ser más sistemática (Grima, op. cit.). Hay constancia de su extracción en la zona de Macael, Lubrín y Cherchos, empleándose en numerosas lápidas, estatuas, columnas o como soportes epigráficos (Padilla, 1999). Se pueden observar ejemplos de esta época en el anfiteatro de Mérida, s. I a.C, (CisnerosCunchillos, 1992), en Itálica (Sevilla), s. III, (BeltránFortes et al., 2011) o incluso en Sagunto (Valencia), s. I, (Sáez-Pérez, op. cit.).

Durante el periodo visigodo se produce un gran parón en lo que se refiere al trabajo de la piedra (Quirós, 1998). Es a partir de la Edad Media, durante el dominio musulmán, cuando comienza la explotación sistemática y planificada de las canteras
(Carretero-Gómez, 1995; Sáez-Pérez, op. cit.; Luque, op. cit.). Los primeros ejemplos en esta época se encuentran en lugares como el palacio de Medina Azahara (Córdoba) (s.X), encargado por Abderramán III con mármoles de Almería, Málaga y Tarragona (Pastor-Medina, op. cit.), el patio de los Leones o la Sala de las Dos Hermanas en la Alhambra (Granada) (s. XIV) entre otras muchas estancias del monumento nazarí. En esta época el mármol de Macael es comercializado con la denominación de "Al Maluki" (EI Real). En el siglo XI, se tiene ya conocimiento de la existencia de una industria de lápidas funerarias que se exportan a lugares tan remotos como a la ciudad nigeriana de Gao (Pastor-Medina, op. cit.).

No obstante, la gran proliferación del uso de este material se produce a partir del s. XVI. La construcción del Monasterio de El Escorial marca un hito en la utilización del mármol en la península. Felipe II quiso dotar a sus conjuntos arquitectónicos de los mejores mármoles y jaspes para embellecer sus fábricas (Sancho, 2004). Así, el marmolista malagueño Juan de Guzmán, con financiación real, recorre todas las sierras andaluzas para localizar los mejores mármoles y jaspes para sus palacios (Cervera, 1985), encontrándose entre ellos los de Macael.

Otro hito en la utilización del mármol en España fue la construcción del Palacio Real de Madrid (s. XVIII). Por razones económicas y para evitar retrasos en las obras, y por el deseo de imitar el ejemplo que los artistas italianos habían dejado dos siglos antes en El Escorial, se ideó, por parte del arquitecto Juan Bautista Saqueti, el primer proyecto de "casamiento de mármoles" para decorar el Palacio (Tárraga-Baldó, 2009). Mediante el empleo de rocas ornamentales hispanas los monarcas pretendieron dar al Palacio Real un marcado carácter nacional, poniendo así de manifiesto la variedad existente en nuestra orografía. En 1787, se desplazó hasta la Sierra de Filabres el marmolista del rey Juan Bautista Galeotti, que escogió para la ornamentación del Palacio la cantera llamada del Pozo y otra junto al río, en el cerro de la Fuente Maestra (Tárraga-Baldó, op. cit.). Así, en el palacio se pueden encontrar ejemplos de uso del mármol de Macael en lugares tan destacados como el Salón delTrono entre otras muchas estancias.

Otros ejemplos importantes del uso del mármol de Macael pueden observarse en diversos edificios de Granada, como el Palacio de Carlos V, la Catedral y la Capilla Real (s. XV), la Catedral de Almería, el Monasterio del Escorial (Madrid) (s. XVI), el Palacio de San Telmo en Sevilla (s. XVII), el Congreso de los Diputados de Madrid (s. XIX) o la Catedral de la Almudena (s. XIX-XX), entre infinidad de iglesias y edificios históricos o actuales (Pastor-Medina, op. cit.). 
En tiempos recientes es uno de los materiales de construcción que gozan de mayor prestigio, tanto nacional como internacional. Algunos de los edificios nacionales más emblemáticos en los que se ha usado el mármol de Macael recientemente son: la Delegación del Gobierno en Granada, la ciudad de la Justicia en Almería, el Centro Islámico de Málaga, el Hospital de Alta Resolución de El Toyo (Retamar, Almería), el edificio de la Seguridad Social en Torrelavega (Cantabria), el Hotel Sorolla Center en Valencia. Internacionalmente destacan el edificio de oficinas Aker Us, en Oslo, el Hotel Crowne Plaza en Yakarta o el prestigioso edificio 396 Alhambra en Miami, entre otros.

En la Fig. 4 se muestran algunos ejemplos del uso, tanto histórico como actual, de los distintos tipos de mármoles extraídos en Macael.

\section{Ensayos de caracterización tecnológica}

\section{Metodología}

Las muestras analizadas han sido facilitadas por la Asociación de Empresarios del Mármol de Andalucía (AEMA), sin que se pueda establecer con precisión las canteras de procedencia. Se han analizado las siguientes variedades: de Blanco Macael las denominadas Rio (BMR), Puntilla (BMPu), Polonia (BMPo) y Cañaílla $(B M C)$ y de Amarillo las variedades Macael Río (AMR), Triana (AT), Indalo (AI) y Alhambra (AA). Además se han analizado otras variedades como Gris Macael (GM) y Anasol (AS).

El análisis mineralógico se ha efectuado por difracción de rayos $X(D R X)$ y microscopía óptica de luz polarizada (MOP). Se ha empleado un Difractómetro X'pert-Pro de Panalytical: $\mathrm{K}$ del $\mathrm{Cu}$, $=1,5405 ; 40 \mathrm{kV}$ de voltaje y $40 \mathrm{~mA}$ de intensidad. Para la identificación de las fases cristalinas se ha empleado el software X-Powder (Martín-Ramos, 2004) y la base de datos PDF-2 (ICDD) para la interpretación de los resultados. Para la caracterización mineralógica y textural del material se ha empleado un microscopio petrográfico de luz polarizada Leica DM2500P con cámara digital incorporada (LEICA DFC450). Mediante estas técnicas se han analizado tan sólo las variedades BMR, AMR, GM y AS, que son las más representativas de cada uno de los litotipos que se extraen actualmente.

Con respecto a las propiedades hídricas se ha determinado la absorción de agua a presión atmosférica (UNE-EN 13755:2008), así como la porosidad abierta, la porosidad total y la densidad aparente (UNE-EN 1936:2007), siguiendo el procedimiento establecido en las normas de referencia.

En cuanto a los parámetros mecánicos, se ha determinado la

- Resistencia a compresión (UNE-EN 1926:2007), que mide la capacidad de un material de soportar cargas elevadas. Mediante este ensayo se determina la resistencia uniaxial no confinada de la roca, o resistencia a compresión simple.

- Resistencia a la flexión bajo carga concentrada (UNE-EN 12372:2007): que mide el esfuerzo que soporta un material sometido a esfuerzos de tracción hasta su rotura. Se ha realizado a tres puntos (carga concentrada).

- Resistencia a los Anclajes (UNE EN13364:2002): mediante este ensayo se determina la fuerza media de carga que una pieza puede soportar antes de fracturarse en los agujeros de anclaje a un paramento. Se aplica una fuerza perpendicular a las caras de la probeta a través de un anclaje colocado en un taladro, en un lateral.

- Resistencia a la Abrasión (UNE-EN 14157: 2005): mide el desgaste en milímetros que se produce en el material que es sometido a rozamiento con un disco de acero y un material abrasivo.

Figura 4. Ejemplos del uso del mármol de Macael: (a) sarcófago fenicio del s. V a.C. (Almagro-Gorbea, op. cit.); (b) anfiteatro romano de Mérida, cuyas columnas son de mármol blanco de Macael (foto: http://jmmlimia.files.wordpress.com/2008/06/img_0064.jpg); (c) estado actual tras la reciente restauración del patio de los Leones en la Alhambra (Granada); (d) altar Mayor de la catedral de Nuestra Señora de la Almudena (Madrid), en la que se emplea el mármol amarillo, junto con serpentinita también de Macael; (e) Hall del edificio empresarial 396 Alhambra (Miami, Florida USA), en el que la solería se ha realizado empleando una combinación de amarillo, gris y blanco de Macael, junto con mármol Negro Marquina de Vizcaya ; (f) edifico Aker Us en Oslo (Noruega), en el que los aplacados de la fachada son de mármol blanco.

Figure 4. Examples of the use of Macael marble: (a) Phoenician sarcophagus. $5^{\text {th }}$ C. B.C. (Almagro-Gorbea, op cit.); (b) Roman amphitheatre in Mérida, whose columns are made of white marble from Macael (photo: http://jmmlimia.files.wordpress.com/ 2008/06/img_0064.jpg); (c) current status after the recent restoration of the Courtyard of the Lions in the Alhambra (Granada); (d) High Altar of the Cathedral of Nuestra Señora de la Almudena (Madrid), in which yellow marble is used along with serpentinite, also from Macael (e) Hall of business building 396 Alhambra (Miami, Florida USA), in which the floor is made using a combination of yellow, grey and white marble from Macael, together with marble Black Marquina from Vizcaya, (f) Aker Us building in Oslo (Norway), where the facade facings are made of white marble. 

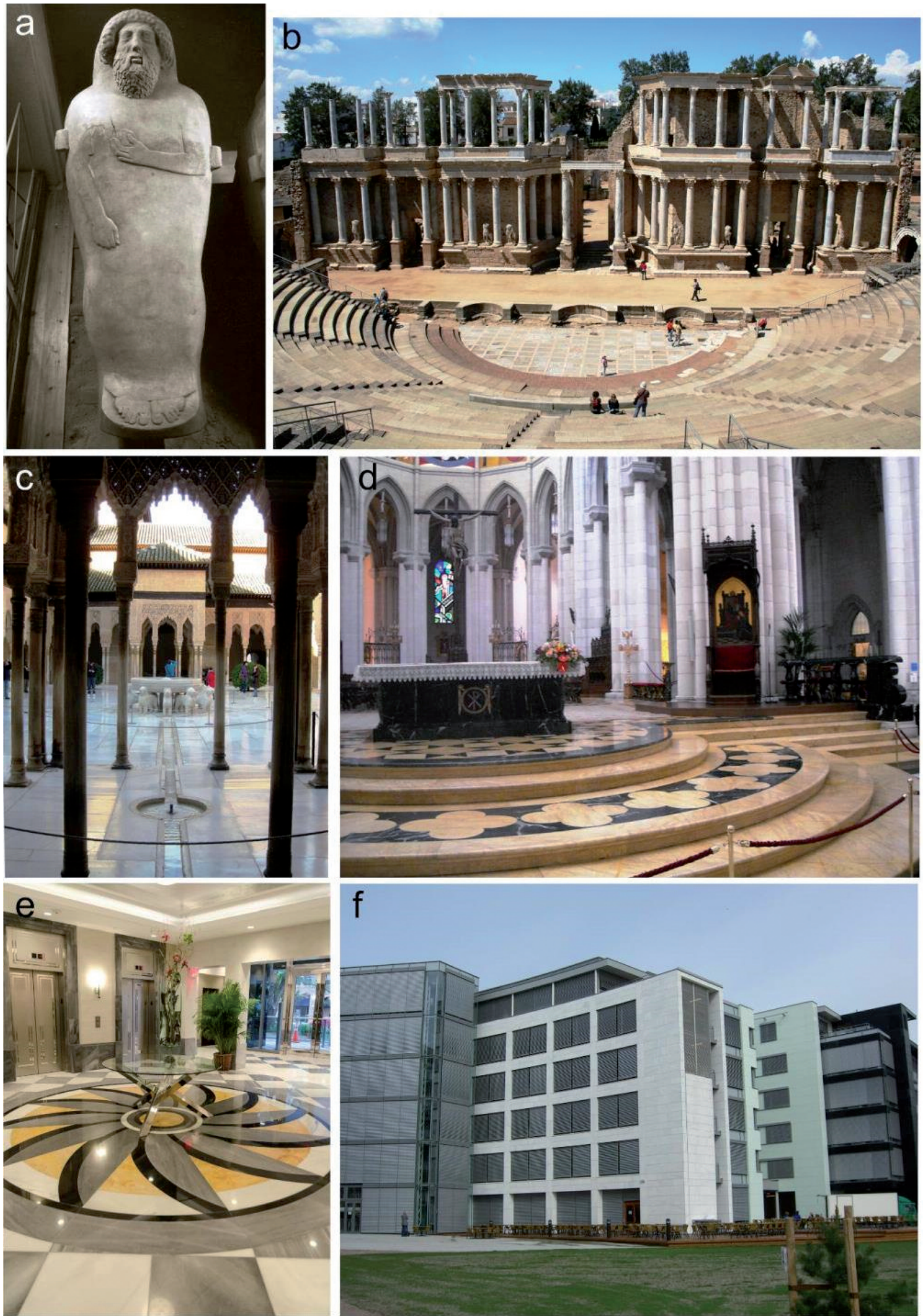
- Resistencia al Deslizamiento (UNE-EN 14231: 2004). Mide la resistencia que ofrece la superficie de la roca a la fricción de una zapata de goma cuando incide sobre la misma por efecto de la caída libre del "brazo" en el que se encuentra situada. Se mide en USRV (Unpolished Slip Resistance Value: valor de la resistencia al deslizamiento de la superficie sin pulido), y va del 0 al 100, siendo 0 la mínima resistencia y 100 la máxima. Se ha evaluado la resistencia que presentan las muestras con los acabados de tipo aserrado, pulido con grano 60 y brillo.

En cuanto a los ensayos de alteración, se han realizado el ensayo de Heladicidad (UNE-EN 12371: 2002), en el que se han realizado un total de 60 ciclos de hielo/deshielo, midiendo posteriormente la resistencia a la flexión de las placas sometidas a estos cambios térmicos. Asimismo, también se ha realizado el ensayo de cristalización de sales (UNE-EN 12370: 1999), que mide la pérdida de masa experimentada por la muestra debido a la degradación que sufre debido a la inmersión en una disolución de sulfato sódico decahidratado $\left(\mathrm{NaSO}_{4} * 10 \mathrm{H}_{2} \mathrm{O}\right)$ diluido al $14 \%$, durante 48 ciclos.

Estos ensayos han sido realizados en el Laboratorio Oficial de Ensayos de Materiales de la Construcción (LOEMCO), siguiendo todos los procedimientos establecidos en las normas UNE de referencia.

\section{Caracterización del Mármol de Macael}

Desde el punto de vista de la mineralogía los mármoles de Macael pueden clasificarse en dos grandes grupos: mármoles calcíticos, en la que la calcita es el mineral predominante, y mármoles dolomíticos, en donde predomina la dolomita. Dentro del primer grupo se han incluido todas las variedades de Blanco, así como el Gris y el Anasol, mientras que en el segundo se encuentras las variedades de Amarillo. En la Fig. 5 se muestra el aspecto macroscópico de muestras pulidas de estas variedades diferenciadas.

Tras la determinación de las fases principales por DRX (Fig. 6) se observa que las muestras de BMR están compuestas principalmente por calcita (99\%), junto con la que aparecen otras fases como micas o apatito; el GM está compuesto también por calcita junto con un porcentaje muy reducido de micas y plagioclasas. La variedad AS, que está compuesta por calcita, junto con la que aparecen anfíboles (enstatita) o micas (flogopita). La variedad de AMR, están compuestas principalmente por dolomita con algo de calcita.

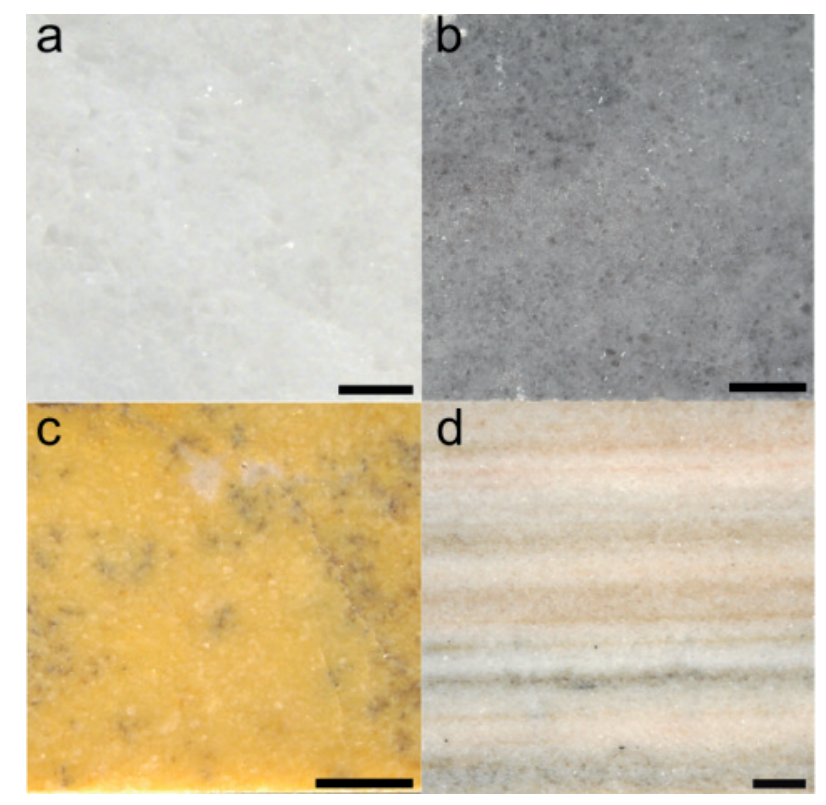

Figura 5. Aspecto macroscópico de muestras pulidas de mármol de Macael: (a) Blanco; (b): Gris; (c) Amarillo; (d) Anasol. Barra de escala: $1 \mathrm{~cm}$.

Figure 5. Macroscopic aspect of polished samples of marble from Macael: (a) White; (b) Grey; (c) Yellow; (d) Anasol. Scale bar: $1 \mathrm{~cm}$.

En cuanto al análisis al MOP, se observa que en el caso del BMR, junto con la calcita aparecen otras fases como cuarzo, micas y minerales opacos, éstos últimos responsable del tenue bandeado negro y gris tan característico que presentan algunas variedades de blanco (Sáez-Pérez, op. cit.). En variedad GM, además de calcita se puede observar algunos granos de cuarzo de forma dispersa junto con micas, plagioclasas y algunos opacos como pirita o ilmenita (Martínez-Martínez, 2008). La variedad AS presenta, además de calcita otros componentes como micas, piroxeno (diópsido), cuarzo y minerales opacos, mientras que la variedad AMR, además de la dolomita presenta calcita, micas, cuarzo y minerales opacos (óxidos de hierro y manganeso).

La textura observada en todos los casos es granoblástica. Las variedades de BMR y GM presentan en general contactos intergranulares rectos y un frecuente maclado. El tamaño de grano de la calcita varía entre los 1.20 y $2.20 \mathrm{~mm}$, aproximadamente (Fig. 7a). En las variedades de AMR (Fig.7b), el tamaño de grano es mucho menor, estando comprendido entre 0.17 y $0.25 \mathrm{~mm}$, presentando además numerosas vetas rellenas de calcita con un tamaño de grano superior o de opacos. Los contactos intergranulares son irregulares y no se observa maclado. La variedad AS tiene un tamaño de grano de la calcita intermedio 


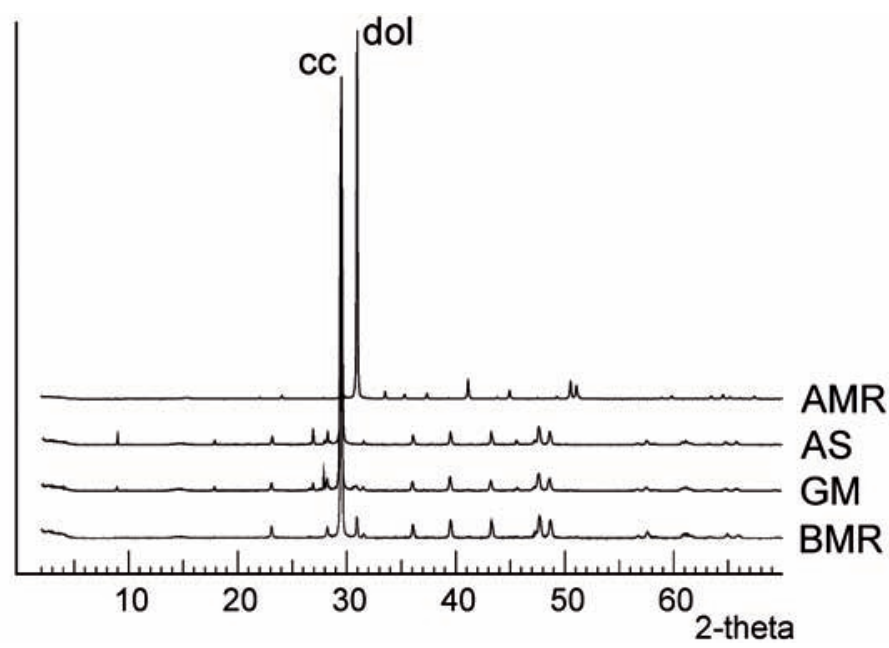

Figura 6. Difractogramas característicos de algunas de las variedades de mármol estudiadas (BMR: Blanco Macael Río; GM: Gris Macael; AS: Anasol; AMR: Amarillo Macael Río; cc: calcita; dol: dolomita).

Figure 6. Diffractograms distinctive of some marble varieties studied (BMR: White Macael Rio; GM: Grey Macael; AS: Anasol; AMR: Yellow Macael Rio; cc: calcite; dol: dolomite).

entre las variedades anteriores, entre 1.00 y $1.90 \mathrm{~mm}$, presentando además contactos rectos y frecuente maclado.

Para el análisis de sus características tecnológicas, se han dividido en dos grupos: mármoles calcíticos y dolomíticos. En la tabla 1 se muestran las principales propiedades físicas y mecánicas así como de alterabilidad de los mármoles estudiados.

En lo referente a las características hídricas de los mármoles calcíticos, se observa que los valores de absorción son bajos en todos los casos, con un rango de valores similar entre todas las variedades. La densidad también es similar y acorde con la mineralogía de las rocas (densidad de la calcita: $2.71 \mathrm{~kg} / \mathrm{m}^{3}$ ). Sin embargo, en la porosidad es donde se producen las variaciones más importantes. La porosidad abierta, es decir, la accesible a los fluidos, presenta un valor significativamente inferior respecto a la porosidad total, lo que es indicativo de que existe poca conexión entre los poros (Andriani y Walsh, 2002). En lo respecta a otras variedades la AS es la que tiene mayor absorción, porosidad abierta y porosidad total con respecto a las variedades de Blanco o de Gris, lo que puede ser indicativo de una mayor fracturación, o bien que las foliación que presente actúe de zonas preferentes de entrada de fluidos.

En cuanto a las propiedades mecánicas, GM es la que mejor comportamiento tiene frente a los esfuerzos de compresión, mientras que BMR es la que menos soporta estos esfuerzos. Ocurre a la inversa con la resistencia a la flexión. Las de mayor valor son AS y BMR, mientras que la de menor valor es la variedad GM. Sucede lo mismo con los valores de resistencia a los anclajes. Respecto a la resistencia a la abrasión todas las muestras presentan valores muy similares, con excepción de las variedades BMR y BMC, que son levemente inferiores. Si se analiza la resistencia al deslizamiento, se observa que la GM es la que presenta valores superiores en los acabados "aserrado" y "grano 60", mientras que es la AS la que mayor resistencia ofrece en el acabado "brillo".

En lo respecta a los ensayos de alteración, se observa que las variedades que mayor pérdida de flexión tras los ciclos del ensayo de heladicidad son las que tenían menor valor inicial en las muestras inalteradas, que son las BMPo y BMC. En cuanto a la resistencia por cristalización de sales, la que mejor comportamiento presenta es la BMR, mientras que BMC es la más susceptible a alteración por este motivo.

De los mármoles dolomíticos, las variedades AMR y AA son las que presentan mayor absorción y mayor porosidad abierta. La porosidad total es en todas las muestras similar excepto en la variedad $\mathrm{Al}$, que es levemente inferior. Estos valores de propiedades hídricas son bastante superiores a los de los mármoles calcíticos. La mayor presencia de fracturas en las variedades dolomíticas va a provocar este incremento en los valores de porosidad. Este hecho va a provocar una mayor facilidad para la penetración de agua y posibles soluciones con sales disueltas, lo que va favorecer los procesos de alteración debido a la formación de sales, a fenómenos de hielo/deshielo o a procesos de disolución (Ruiz-Agudo et al. 2008).

En cuanto al comportamiento mecánico de los mármoles dolomíticos se observa que las variedades AT y Al, que son las que menor porosidad abierta y absorción presentan, son las que presentan una mayor resistencia a la compresión. Sin embargo esta relación no se cumple para el resto de propiedades mecánicas. La variedad AT es la que mayor resistencia a la tracción presenta, y sin embargo ofrece menor resistencia a los anclajes que el resto, mientras que con la variedad AA ocurre justo a la inversa. La variedad AT es la que presenta una mayor resistencia a la abrasión, al igual que a resistencia al deslizamiento en el acabado "aserrado" o "brillo", mientras que en el acabado "grano 60", todas las muestras se comportan igual. En conjunto, en relación con los mármoles calcíticos, las variedades dolomíticas presentan una mayor resistencia a la compresión, a pesar de tener una mayor porosidad (Martínez-Martínez, op. cit.). En este caso el menor tamaño de grano de las variedades dolomíti- 

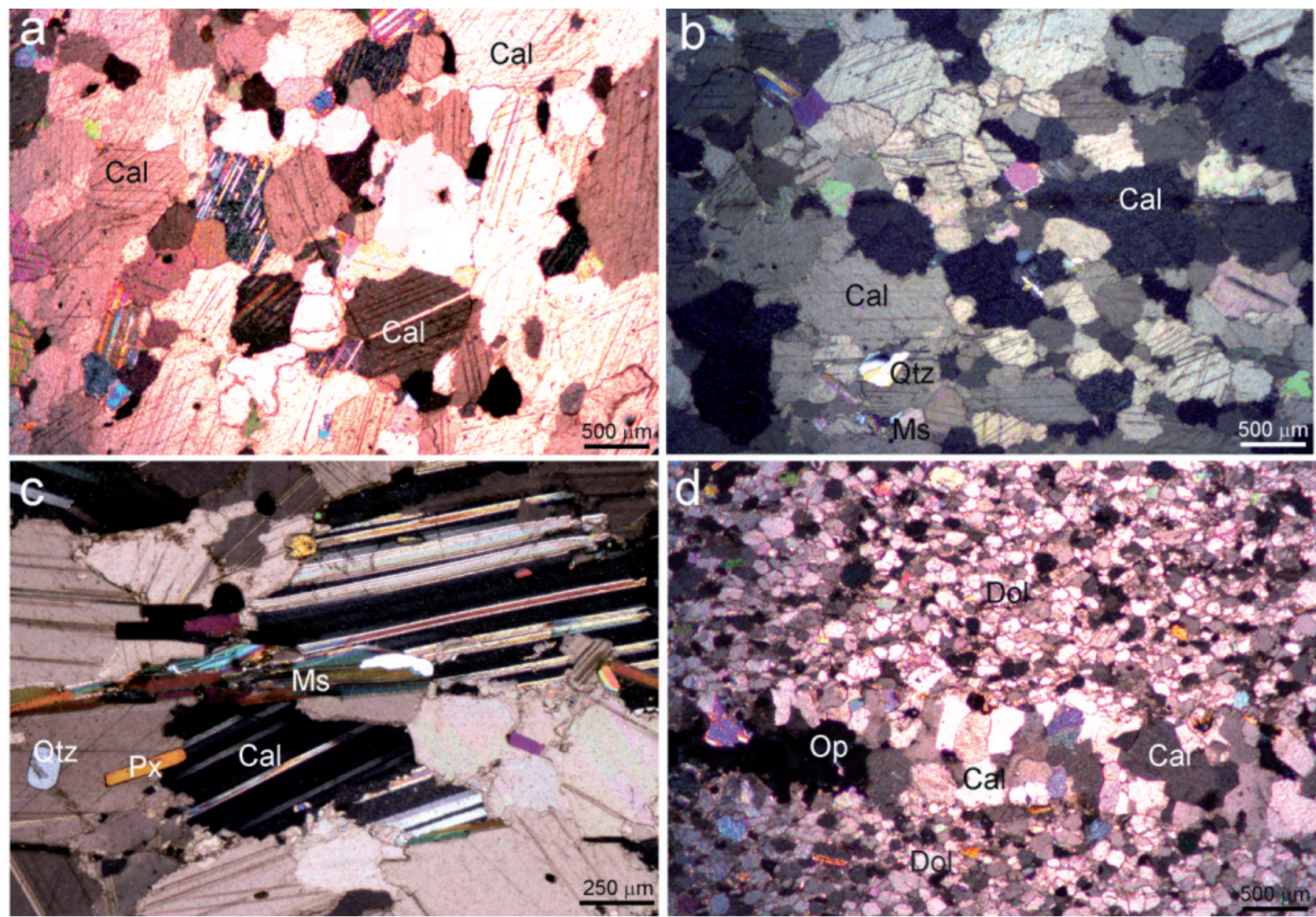

Figura 7. Microfotografías con nícoles cruzados de (a) Blanco Macael Río (calcítico); (b) Gris Macael (Calcítico); (c) Anasol (calcítico); (d) Amarillo Macael Río (dolomítico), en la que se observa una veta rellena de calcita y minerales opacos. Abreviaturas: Cal: calcita; Dol: dolomita; Ms: moscovita; Op: mineral opaco; Px: Piroxeno; Otz: cuarzo.

Figure 7. Photomicrographs with crossed nicols of: (a) White Macael Río (calcitic); (b) Grey Macael (calcitic); (c) Anasol (calcitic); (d) Yellow Macael Río (dolomitic) in which a vein filled with calcite and opaque minerals is observed. Abbreviations: Cal: calcite; Dol: dolomite; Ms: muscovite; Op: opaque mineral; Px: Pyroxene; Otz: quartz.

cas se estima como un aspecto fundamental de cara al incremento de la resistencia a la compresión (Hatzor y Palchik, 1998). Asimismo se observa una similar resistencia a la flexión, aunque algo inferior en los dolomíticos, y una menor resistencia a los anclajes. La resistencia a la abrasión es también algo superior a los calcíticos, mientras que la resistencia al deslizamiento presenta valores similares en ambos casos, algo superior en el acabado "brillo" para los dolomíticos.

Tras el ensayo de heladicidad se observa el mismo fenómeno que ocurría con los mármoles calcíticos, es decir las muestras inalteradas con menores valores de resistencia a la flexión, son las que más pérdida sufre tras el ensayo de alteración, destacando la variedad $A A$ que sufre una pérdida del $75.8 \%$. En cuanto a la pérdida de peso por cristalización de sales, es la variedad AMR la que peor se comporta, aunque todos los valores son muy similares. El deterioro por cristalización de sales en el interior de un sólido poroso se ve favorecido por la porosidad total, la distribución del tamaño de poros y el tipo de poro (Scherer, 1999 y Valenza y Scherer, 1996) y por la composición de la roca (Li et al., 2008). Los mármoles dolomíticos, a pesar de tener una mayor porosidad y absorción que los calcíticos, y por tanto una mayor facilidad para que penetren fluidos ricos en sales (Rodríguez-Navarro y Doehne, 1999), se comportan mejor frente al ataque por sales. Este hecho es debido a que, a pesar de que tanto la calcita como la dolomita tienen valores de solubilidad similares en $\mathrm{NaSO}_{4}$, la velocidad de disolución es entre 3 y 60 veces menor en la dolomita que en la calcita (Martínez-Martínez, op. cit.), por lo que las variedades calcíticas van a ser mucho más susceptibles de alteración por efecto de las sales. 
Navarro, R., et al., 2017. Caracterización de los principales tipos de mármol .... Boletín Geológico y Minero, 128 (2): $345-361$

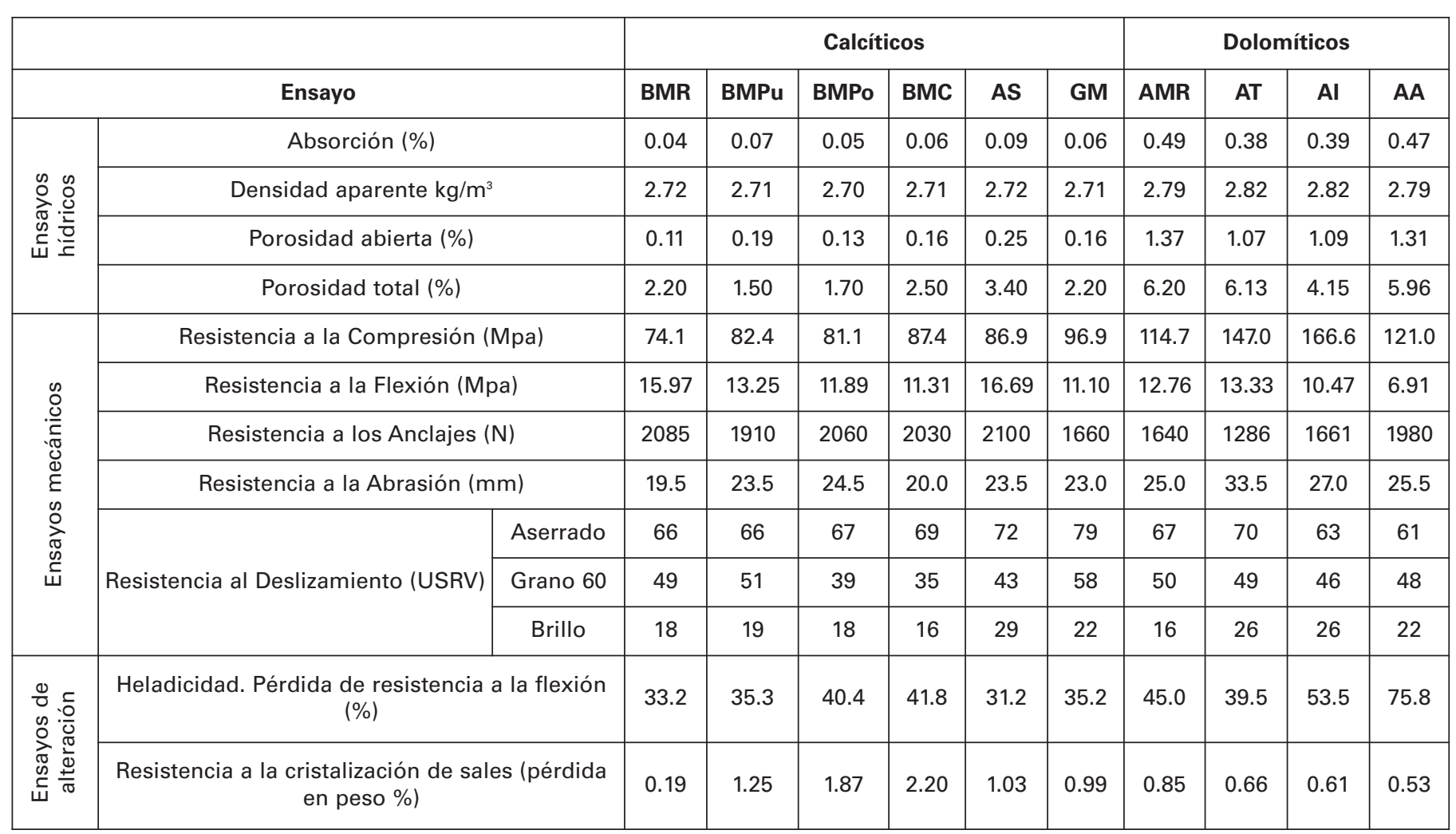

Tabla 1. Principales características tecnológicas de las variedades del mármol de Macael: Blanco Macael Rio (BMR), Blanco Macael Puntilla (BMPu), Blanco Macael Polonia (BMPo), Blanco Macael Cañaílla (BMC), Gris Macael (GM) Anasol (AS), Amarillo Macael Río (AMR), Amarillo Triana (AT), Amarillo Indalo (Al) y Amarillo Alhambra (AA).

Table 1. Main technological features of different varieties of marble from Macael: White Macael Río (BMR), White Macael Puntilla (BMPu), White Macael Polonia (BMPo), White Macael Cañaíla (BMC), Grey Macael (GM) Anasol (AS), Yellow Macael Río (AMR), Yellow Triana (AT), Yellow Indalo (Al) and Yellow Alhambra (AA).

\section{Análisis del sector}

En el año 2011, la producción mundial de piedra natural fue de unos $116 \mathrm{Mt}$ de los que $68.5 \mathrm{Mt}$ (59\%) corresponden al mármol, travertino y piedra caliza (AIDICO, 2012). La explotación y manufactura de rocas ornamentales en España es una de las actividades mineras de mayor relevancia en el sector minero. De hecho, España, actualmente es el séptimo productor mundial de piedra natural tras Italia y Brasil, con una producción en el año 2011 de $5.5 \mathrm{Mt}$ (AIDICO, op. cit.), de las que $2.3 \mathrm{Mt}$ corresponden a calizas y mármoles ornamentales (INE, 2013).

La mayor parte de estas explotaciones de calizas y mármoles se concentran en el Sureste peninsular, destacando por la calidad de los materiales las zonas productoras de Pinoso-Novelda (Alicante), con 1.05 Mt producidas en 2011, Caravaca-Cehegín (Murcia), con 410000 t y Macael (Almería), con 210000 t (INE, op. cit.). Esto supone que en el sureste español se produce el $72 \%$ de toda la producción nacional. De estas zonas, el mármol de la comarca de Macael es, sin lugar a dudas, el de mayor reconocimiento a nivel tanto nacional como internacional, tanto por su uso en la industria de la construcción actual, nacional e internacional, como histórico. Esto ha sido consecuencia de los diferentes planes de desarrollo impulsados en la región en los últimos 30 años, que ha hecho que se pase de ser una industria dedicada tan solo a la extracción a realizar todas las fases del proceso productivo, otorgándole al producto un importante valor añadido, además de realizar una importante política comercializadoras, tanto en el mercado interior como en el exterior (Carretero-Gómez y Aznar-Sánchez, 2012).

A pesar de la caída del sector en los últimos años que ha supuesto una gran reducción en el número de explotaciones activas de la región, la fuerte demanda internacional de este producto hace que mantenga aún unos altos índices de productividad respecto a otros sectores de la construcción. Según datos de la Asociación de Empresarios del Mármol de Andalucía 
Navarro, R., et al., 2017. Caracterización de los principales tipos de mármol .... Boletín Geológico y Minero, 128 (2): $345-361$

(AEMA) en el año 2012 permanecían activas 40 canteras habiéndose producido el cierre del $60 \%$ de las explotaciones mineras en el periodo 2008-2012. Esto ha supuesto el cese de actividad del $15 \%$ de las empresas del sector en ese periodo. Esto ha sido consecuencia de una bajada en las ventas en el mercado nacional de un $60 \%$. Actualmente se contabilizan un total de 45 empresas dedicadas a la extracción y 105 de tratamiento y comercialización y 60 empresas dedicadas a la artesanía, aunque si se tiene en cuenta que algunas empresas se dedican tanto a la extracción, como a la elaboración, el número se reduce a 120 empresas. Esta industria dio empleo de forma directa a un total 3250 trabajadores, cifra muy alejada de los 5600 trabajadores que llegó a tener el sector en el año 2006.

En la tabla 2 se muestra la variación de la producción en fábrica en los 10 últimos años, así como las exportaciones.

Destaca como la producción ha caído casi a la mitad en estos años. Sin embargo las empresas, ante la caída del mercado nacional, optaron por potenciar el comercio exterior, hecho que se ha visto recompensado con un incremento paulatino del volumen de exportaciones, destacando el año 2012 con un incremento de casi el 50\% respecto al año anterior. Los principales países a los que se exporta el mármol de Macael son Estados Unidos, Alemania, Reino Unido, Países Bajos, Francia, Irlanda, Portugal, Italia, Japón, Marruecos, Emiratos Árabes, Rusia o China.

\section{Conclusiones}

El mármol de Macael es una de las rocas ornamentales más antiguas empleadas en la Península lbérica. A pesar de haber indicios de extracción más o menos importantes desde hace más de 4000 años, son los árabes los que sistematizan su extracción a partir del s. XI. Desde entonces es una de las rocas ornamentales más valoradas en construcción. Se pueden encontrar ejemplos de su uso en monumentos por toda la Península Ibérica, bien como elementos estructurales o como decorativos, tanto en interiores o en exteriores. Algunos de relevancia como el Monasterio de EI Escorial, el Palacio Real o el Patio de los Leones de la Alhambra, en Granada. También en los últimos años es un material muy demandado internacionalmente, hecho que se manifiesta en un incremento de las exportaciones de cerca del 50\% en el año 2012 respecto al año anterior.

Las variedades comerciales más características del mármol de Macael incluyen tanto mármoles calcíticos como dolomíticos, y son las conocidas genéricamente como Blanco Macael, Amarillo Macael, Gris Macael y Anasol. La mayor parte de los mármoles se extraen de la formación Las Casas, que pertenece a la Unidad Bédar-Macael (Unidad Superior del CNF, Zonas Internas de las Cordilleras Béticas).

Las variedades de Blanco Macael, Gris y Anasol están compuestas principalmente por calcita, junto con otros minerales como micas, cuarzo, piroxenos, plagioclasas y minerales opacos (pirita, ilmenita) como accesorios, mientras que las variedades de Amarillo están formados por dolomita, junto con la que además se observan, en un porcentaje mucho menor otras fases como calcita, micas, cuarzo y óxidos de hierro y manganeso.

En lo que respecta a sus propiedades, se observa que los mármoles calcíticos presentan porosidad, absorción y densidad más bajos que los dolomíticos, mientras que los mármoles dolomíticos presentan

\begin{tabular}{|c|c|c|c|c|c|c|c|c|c|c|}
\hline & 2003 & 2004 & 2005 & 2006 & 2007 & 2008 & 2009 & 2010 & 2011 & 2012 \\
\hline $\begin{array}{l}\text { Ventas estimadas } \\
\text { (millones de euros) }\end{array}$ & 661.11 & 710.48 & 761.23 & 751.36 & 689.87 & 524.80 & 445.00 & 430.00 & 425.00 & 470.00 \\
\hline $\mathrm{n}^{\circ}$ trabajadores & 5300 & 5400 & 5520 & 5600 & 5564 & 3559 & 5545 & 4311 & 3169 & 3250 \\
\hline $\begin{array}{l}\text { Producciones en fábrica } \\
\qquad\left(\text { miles } \mathrm{m}^{2}\right)\end{array}$ & 21283 & 21507 & 21639 & 21760 & 19370 & 19435 & 15058 & 12860 & 12748 & 12810 \\
\hline Variación (\%) & & 5.4 & -21.1 & 1.6 & 0.3 & 10.9 & -18.1 & 1.1 & 2.7 & 49.2 \\
\hline
\end{tabular}

Tabla 2. Principales indicativos del estado del sector del mármol en Macael durante el periodo 2003-2012 (Fuente elaboración propia con datos de AEMA).

Table 2. Main indicators of the state of the marble industry in Macael during the period 2003-2012 (Source: Own elaboration with data of AEMA). 
mayor resistencia a la compresión, una resistencia a la flexión similar, algo inferior, y una resistencia a los anclajes inferior que los calcíticos. Los valores de resistencia a la abrasión son también mejores en los dolomíticos, mientras que la resistencia al deslizamiento es similar en todos los casos. En cuanto a la alteración los dolomíticos, con la excepción de la variedad AT, se alteran más en los ciclos de hielo/deshielo. En cuanto a la alteración por cristalización de sales, la que menos alteración presenta es la variedad BMR (calcítica), pero en conjunto, los mármoles dolomíticos presentan menos alteración que los calcíticos.

$\mathrm{El}$ adecuado conocimiento de las características tecnológicas de este material y el estado actual del sector permitirá optimizar su empleo tanto en obra nueva como restauraciones y establecer nuevas estrategias para poner aún más en valor uno de los materiales más importantes en la historia de la construcción de nuestro país.

\section{Agradecimientos}

Este trabajo ha sido financiado a través de CONSTRUROCK (Red de la Piedra Natural y su relación con el Patrimonio Histórico-Monumental y la obra nueva, en el ámbito del Estado español) y de la Asociación de Empresarios del Mármol de Macael (AEMA).

Los autores también quieren agradecer a dos revisores anónimos sus correcciones y sugerencias para ayudar a mejorar este artículo.

\section{Referencias}

AIDICO. 2012. Informe sectorial de la piedra natural en España 2011. Observatorio del mercado de la Piedra Natural. Instituto Tecnológico de la Construcción, Valencia, 74 pp.

Almagro-Gorbea, M., López-Rosendo, M.E., MederosMartín, A. y Torres-Ortiz, M. 2010. Los sarcófagos antropoides de la necrópolis de Cádiz. Mainake, XXXII (I), 357394.

Andriani, G. F. y Walsh, N. 2002. Physical properties and textural parameters of calcarenitic rocks: qualitative and quantitative evaluations. Engineering Geology, 67, 5-15.

Baltuille, J.M., Gisbert, J., Pereria, M.D., Sebastián, E., Mota, M.I., Gómez-Gras, D., Taboada, J., García de los Ríos, A., Franco, A. y Fort, R. 2012. CONSTRUROCK: a network at the service of natural stone and the architectonic heritage. Global Stone Congress, Borba, Alentejo (Portugal), 4 pp.

Bello, M.A., Martin, L. y Martin, A. 1992. Microchemical identification of Macael White marble in some Spanish monuments. Materiales de Construcción, 225, 23-30.

Beltrán-Fortes, J., Loza-Azuaga, L., Ontiveros-Ortega, E.,
Rodríguez-Gutiérrez, O. y Taylor, R. 2011. La explotación y el empleo de marmora en la baetica. Un proyecto de investigación de base arqueométrica. Itálica. Revista de arqueología clásica de Andalucía, 1, 51-75.

Benavente, D. 2006. Propiedades físicas y utilización de rocas ornamentales. En: García del Cura, M. A. y Cañaveras, J. C. (eds.), Utilización de rocas y minerales industriales. Seminarios SEM, Alicante, 123-153.

Carretero-Gómez, A. 1995. La industria del mármol en Almería. Servicio de Publicaciones de la Universidad de Almería, Almería, 383 pp.

Carretero-Gómez, A. y Aznar-Sánchez, J.A. 2012. La política de desarrollo local en la comarca del mármol en Almería Revista de Estudios Empresariales. Segunda época, 1, 113-130.

Cervera, L. 1985. Años del primer matrimonio de Juan de Herrera. Albatros, Valencia, 380 pp.

Cisneros-Cunchillos, M. 1992. La explotación de los mármoles almerienses en la época romana. Roc Máquina, 6, 4447.

Cooper, B.J., Marker, B.R., Pereira, M.D. y Schouenborg, B. 2013. Establishment of the "Heritage Stone Task Group" (HSTG). Episodes, 36 (1), 8-10.

Doehne, E. y Price, C.A. 2010. Stone conservation. An overview of current research (2nd Edition). The Getty Conservation Institute, Los Angeles, California, 174 pp.

Egeler, C. G. 1963. On the tectonics of the eastern Betic Cordilleras (SE Spain). Geologische Rundschau, 52, 260269.

García-Dueñas, V., Martínez-Martínez, J.M., Orozco, M. y Soto, J.I. 1988. Plis-nappes, cisillements syn- à postmétamorphiques et cisaillements ductiles-fragiles en distension dans les Nevado-Filabrides (Cordillères bétiques, Espagne). Comptes Rendus de l'Académie des Sciences de Paris. Série II, 307, 1389-1395.

Grima, J. 1994. La explotación del mármol de Macael y de los Filabres a lo largo de la historia (I): Desde la antigüedad hasta el fin del periodo nazarita. En: Grima, J. y Martínez-Cosentino, F. (ed.), La Piedra Natural. Su papel en la historia. Nuevo reto de la minería y la industria en España. Arráez Editores, Almería, 27-42.

Harrell, J. A., 2003. Survey of ornamental stones in mosques and other Islamic buildings of the Pre-Ottoman Period in Cairo, Egypt, Department of Environmental Science. Universidad of Toledo (Ohio), USA., 11/23/11, http://www.eeescience.utoledo.edu/faculty/harrell/Egypt /Mosques/Survey_Intro.htm.

Hatzor, Y. H. y Palchik, V. 1998. A microstructure-based failure criterion for Aminadav dolomites. International Journal of Rock Mechanics and Mining Sciences and Geomechanics Abstracts, 35 (6), 797-805.

INE. 2013. Estadística Minera de España 2011, Instituto Nacional de Estadística. Ministerio de Industria, Energía y Turismo, 11/11/13, http://www.minetur.gob.es/ENERGIA/MINERIA/ESTADISTICA/Paginas/EstadisticaMinera. aspx

Kampschuur, W., Vissers, R., García-Monzón, G. y de las Heras, A. 1975. Mapa geológico de España Escala 1:50000. Hoja 1013: Macael. Mapas y Memoria. IGME, Madrid. 
Navarro, R., et al., 2017. Caracterización de los principales tipos de mármol .... Boletín Geológico y Minero, 128 (2): $345-361$

Li, L., Steefel, C. I. yYang, L 2008. Scale dependence of mineral dissolution rates within single pores and fractures. Geochimica et Cosmochimica Acta, 72, 360-377.

López-Jimeno, C. 1995. Manual de rocas ornamentales: prospección, explotación, elaboración, colocación. Entorno Gráfico, Madrid, 728 pp.

Luque, A. 2010. Andalucian Marbles: Durability criteria applied in tis use as ornamental stone. PhD. Universidad de Granada. 219 pp.

Luque, A., Leiss, B., Álvarez-Lloret, P., Cultrone, G., Siegesmund, S., Sebastián-Pardo, E. y Cardell, C. 2011a. Potential thermal expansion of calcitic and dolomitic marbles from Andalusia (Spain). Journal of Applied Crystallography, 44, 1227-1237.

Luque, A., Martínez deYuso, M. V., Cultrone, G. y SebastiánPardo, E. 2013. Analysis of the surface of different marbles by X-ray Photoelectron Spectroscopy (XPS) to evaluate decay by $\mathrm{SO}_{2}$ attack. Environmental Earth Sciences, 68, 833-845.

Luque, A., Ruiz-Agudo, E., Cultrone, G., Sebastián-Pardo, E. y Siegesmund, S. 2011 b. Direct observation of microcrack development in marble caused by thermal weathering. Environmental Earth Sciences, 62 (7), 1375-1386

Martín-Algarra, A.C., Alonso-Chaves, F.M., Andreo, B., Azañón, J.M., Balanyá, J.C., Booth-Rea, G., CrespoBlanc, A., Delgado, F., Díaz de Federico, A., Estévez, A., Galindo-Zaldívar, J., García-Casco, A., García-Dueñas, V., Garrido, C.J., Gervilla, F., González-Lodeiro, F., Jabaloy, A., López-Garrido, A.C., Martín-Algarra, A., MartínMartín, M., Nieto, J.M., O’Dogherty, L., Orozco, M., Puga, E., Rodríguez-Cañero, R., Ruiz-Cruz, M.D., SánchezGómez, A., Sánchez-Navas, A., Sanz de Galdeano, C., Soto, J. I., Torres-Roldán, R.L. y Vera, J. A. 2004. Zonas Internas Béticas. En: Vera, J.A. (ed.), Geología de España. SGE-IGME, Madrid, 395-444.

Martínez-Martínez, J. 2008. Influencia de la alteración sobre las propiedades mecánicas de calizas, dolomías y mármoles: evaluación mediante estimadores no destructivos (ultrasonidos). Tesis Doctoral. Universidad de Alicante. 295 pp.

Martínez-Martínez, J.M., Soto, J.I. y Balanyá, J.C. 2002. Orthogonal folding of extensional detachments: Structure and origin of the Sierra Nevada elongated dome (Betics, SE Spain). Tectonics, 21 (3), 1-20.

Martín-Ramos, J.D. 2004. Using XPowder`C, A Software Package for Powder X-Ray Diffraction Analysis D.L.GR1001/04, Spain, 1-105.

Navarro, R., Pereira, M.D., Gimeno, A. y Del Barrio, S. 2013. Verde Macael: A serpentinite wrongly referred to as a marble. Geosciences, 3 (1), 102-113.

Padilla, A. 1999. Consideraciones en torno a la explotación del mármol en la Bética durante los s. I-II. Habis, 271-281.

Pastor-Medina, G. 1990. Macael morisco y cristiano. Instituto de estudios almerienses. Ayuntamiento de Macael, Almería, 125 pp.

Pereira, M.D., Peinado, M. y Blanco, J.A. 2013. Misuse of natural stone for construction and the consequences in buildings. Case of study of serpentinites. Journal of Materials in Civil Engineering, 25 (10), 1563-1567.

Quirós, J. A. 1998. La sillería y las técnicas constructivas medievales: Historia social y técnica de la producción arquitectónica. Archeologia Medievale, 25, 235-246.

Rodríguez-Gordillo, J. y Sáez-Pérez, M. P. 2006. Effects of thermal changes on Macael marble: Experimental study. Construction and Building Materials, 20, 355-365.

Rodríguez-Gordillo, J. y Sáez Pérez, M. P. 2010. Comportamiento físico del mármol blanco de Macael (España) por oscilación térmica de bajo y medio rango. Materiales de Construcción, 60 (297), 127-141.

Rodríguez-Navarro, C. y Doehne, E. 1999. Salt weathering: influence of evaporation rate, supersaturation and crystallization pattern. Earth Surface Processes and Landforms, 24 (3), 191-209.

Ruiz-Agudo, E., Putnis, C. V. y Rodriguez-Navarro, C. 2008. Interaction between epsomite crystals and organic additives. Crystal Growth \& Design, 8, 2665-2673.

Sáez-Pérez, M.P. 2004. Estudio de elementos arquitectónicos y composición de materiales del Patio de los Leones. Interacciones en sus causas de deterioro. Servicio de publicaciones de la Universidad de Granada, Granada, 429 pp.

Sáez-Pérez, M.P. y Rodríguez-Gordillo, J. 2009. Structural and compositional anisotropy in Macael marble (Spain) by ultrasonic, XRD and optical microscopy methods. Construction and Building Materials, 23 (6), 2121-2126.

Sancho, J.L. 2004. El Palacio Real de Madrid. Patrimonio Nacional, Madrid, 256 pp.

Scherer, G. W. 1999. Crystallization in pores. Cement and Concrete Research, 29, 1347-1358.

Siret, E. y Siret, L. 2006. Las primeras edades del metal en el sudeste de España. Álbum. Edición Facsimilar. Dirección General de Cultura. Museo Arqueológico de Murcia, Murcia, 182 pp.

Tárraga-Baldó, M.L. 2009. Mármoles y rocas ornamentales en la decoración del Palacio Real de Madrid. Archivo Español de Arte, LXXXII (328), 367-392.

UNE-EN-1926. 2007. Métodos de ensayo para la piedra natural. Determinación de la resistencia a la compresión uniaxial. A.E.N.O.R., Madrid, 22 pp.

UNE-EN-1936. 2007. Métodos de ensayo para piedra natural. Determinación de la densidad real y aparente y de la porosidad abierta y total. A.E.N.O.R., Madrid, 13 pp.

UNE-EN-12370. 1999. Métodos de ensayo para piedra natural. Determinación de la resistencia a la cristalización de las sales. A.E.N.O.R., Madrid, 12 pp.

UNE-EN-12371. 2002. Métodos de ensayo para piedra natural. Determinación de la resistencia a la heladicidad. A.E.N.O.R., Madrid, 18 pp.

UNE-EN-12372. 2007. Métodos de ensayo para piedra natural. Determinación de la resistencia a la flexión bajo carga concentrada. A.E.N.O.R., 20 pp.

UNE-EN-13364. 2002. Métodos de ensayo para piedra natural. Determinación de la carga de rotura para anclajes. A.E.N.O.R., Madrid, 20 pp.

UNE-EN-13755. 2008. Métodos de ensayo para piedra natural. Determinación de la absorción de agua a presión atmosférica. A.E.N.O.R., Madrid, 12 pp.

UNE-EN-14157. 2005. Métodos de ensayo para piedra natural. Determinación de la resistencia a la abrasión. A.E.N.O.R., Madrid, 22 pp. 
UNE-EN-14231. 2004. Métodos de ensayo para piedra natural. Determinación de la resistencia al deslizamiento mediante el péndulo de fricción. A.E.N.O.R., Madrid, 18 pp.
Valenza, J. J. y Scherer, G. W. 2006. Mechanism for salt scaling. Journal of the American Ceramic Society, 89 (4), 1161-1179.

Recibido: diciembre 2015

Revisado: febrero 2016

Aceptado: abril 2016

Publicado: junio 2017 
